\title{
El barómetro cultural local (báculo): una herramienta para el seguimiento y la evaluación de las políticas culturales locales
}

\author{
Vicente Coll-Serrano \\ Doctor en Economía (Universidad de Valencia); Profesor de la Universidad de Valencia. \\ Valencia, Espanha \\ vicente.coll@uv.es \\ Luis Vila-Lladosa \\ Doctor en Economía (Universidad de Valencia); Profesor de la Universidad de Valencia. \\ Valencia, Espanha \\ luis.vila@uv.es \\ Olga Blasco-Blasco \\ Doctora en Economía (Universidad de Valencia); Profesora de la Universidad de Valencia. \\ Valencia, Espanha \\ olga.blasco@uv.es \\ Salvador Carrasco-Arroyo \\ Doctor en Economía (Universidad de Valencia); Profesor de la Universidad de Valencia. \\ Valencia, Espanha \\ salvador.carrasco@uv.es
}

\begin{abstract}
Resumen El Barómetro Cultural Local (Báculo) es una herramienta que proporciona información a los gobiernos locales para apoyar tanto los procesos de planificación y toma de decisiones como el seguimiento y evaluación de las políticas culturales. A partir de los datos contenidos en el Sistema de Indicadores Culturales Local (SICLO), la información que ofrece Báculo a los usuarios se sintetiza en tres indicadores multidimensionales: Sintonía, Acción e Impulso-Respuesta. En este trabajo se describen las principales características de Báculo, es decir, cómo se obtienen los indicadores que lo integran y cómo se muestra la información a los gobiernos locales.
\end{abstract}

Palabras clave: Sistema de Indicadores, indicadores culturales, política cultural, gobierno local y municipal, planificación, toma de decisiones.

\section{Introducción}

Z $\mathrm{n}$ España, la Federación Española de Municipios y Provincias ¿ (FEMP) es la Asociación de Entidades Locales de ámbito estatal con mayor implantación, agrupando a un total de 7.324 entidades entre Ayuntamientos, Diputaciones, Consejos y Cabildos Insulares, lo que equivale a una representación de más del 90\% de los Gobiernos Locales españoles.

La FEMP se estructura en cinco direcciones generales: Comunicación; Organización y Recursos, Servicios Jurídicos y Coordinación Territorial, Estudios y Formación y Políticas Locales. Esta última, a su vez, se encuentra integrada por el Área Económica y las subdirecciones 
generales de: Modernización de la Administración, Asuntos Sociales, Educación y Cultura, Desarrollo Sostenible y, por último, Movilidad y Territorio.

Dentro de la Subdirección general de Educación y Cultura, el Área de Cultura tiene como objetivo principal la construcción de herramientas útiles para la planificación cultural y la evaluación de las políticas culturales locales, así como la puesta en común de buenas prácticas.

En el ámbito de lo público, y en un contexto cada vez más complejo y cambiante que describe modelos efectivos de intervención sobre la configuración de los espacios simbólicos (Evans, 2001), la planificación debe entenderse como una herramienta imprescindible para la identificación de prioridades y la asignación de recursos. Desde esta perspectiva, la planificación cultural marca los límites desde los cuales se fijan los procesos de seguimiento y evaluación del desempeño de las administraciones en general, y de las políticas públicas culturales en particular.

Los procesos de seguimiento y evaluación de los planes, programas y proyectos se fundamentan en otros procesos relacionados con la observación de la realidad cultural. Esto obliga a la sistematización de la recogida de datos, su almacenamiento y tratamiento para proporcionar los elementos y resultados del análisis de la información, imprescindibles para la toma de decisiones. Este proceso, encaminado a producir un cambio, se enmarca en lo que podemos denominar un Sistema Integrado de Información Cultural (SIIC) (Carrasco, 2006).

Disponer de un SIIC robusto y fiable es condición necesaria para conocer y evaluar las dinámicas existentes o emergentes en el territorio, así como para diseñar e implementar un Sistema de Indicadores Culturales. En este estudio se presentan las principales acciones que se han llevado a término con la finalidad de hacer efectivamente operativo el sistema de indicadores diseñado por la FEMP (FEMP, 2009) para apoyar la necesaria evaluación de las políticas culturales de ámbito local (Martinell, 2011; Mussot, 2011). El resultado final del trabajo realizado se ha materializado en la construcción de una herramienta orientada tanto a apoyar los procesos de planificación y toma de decisiones como el seguimiento y evaluación de las políticas culturales de los gobiernos locales. Esta herramienta, que recibe el nombre de Barómetro Cultural Local (BÁCULO), es versátil y fácilmente reproducible en otros escenarios.

El resto del trabajo se estructura como sigue. En el apartado 2 se revisan brevemente los antecedentes del Sistema de Indicadores para la evaluación de las políticas culturales de la FEMP. En el apartado 3 se describen las principales características de la herramienta BÁCULO, sus componentes y funcionalidades. Por último, el apartado 4 se dedica a las conclusiones.

\section{Antecedentes}

A principios del año 2005, la Comisión de Cultura de la FEMP designó un grupo técnico, constituido por los responsables de diversas agencias locales especializadas en la gestión de información cultural y evaluación de políticas culturales, con la finalidad de (Escudero, 2009): (i) describir la situación de los sistemas de información cultural local existentes en aquel momento en España, (ii) intentar transmitir las necesidades de información existentes a nivel local a los organismos con responsabilidad en cada campo (Ministerio de Cultura, Instituto Nacional de Estadística, etc.), (iii) generar instrumentos para la evaluación de las políticas locales para la cultura y (iv) contribuir a crear condiciones para el trabajo en red entre las agencias locales especializadas existentes.

Desde un principio, el grupo técnico de la FEMP contó con la colaboración de la Dirección General de Cooperación y Comunicación Cultural del Ministerio de Cultura. ${ }^{1}$ Esta colaboración se tradujo en la firma de sucesivos convenios entre el Ministerio de Cultura y la FEMP, convenios que secuencialmente fueron dando cobertura a las distintas fases del proyecto.

En marzo de 2006, el grupo técnico decidió seleccionar un grupo más reducido de trabajo de entre sus miembros, al que se le asignó la responsabilidad de construir un sistema de indicadores basado en la necesidad de (i) orientar las políticas culturales locales hacia las tesis planteadas en la Agenda 21 de la Cultura (CGLU, 2004) e (ii) implantar sistemas de información que permitan realizar una planificación estratégica en la aplicación de la política cultural de las administraciones públicas. Para acometer el proyecto encomendado este equipo de trabajo contaba con la ayuda de una empresa consultora especializada.

El sistema de indicadores que se perseguía construir, como se ha comentado en el párrafo anterior, debía articularse en torno a los principios y recomendaciones que contiene la Ag21 de la Cultura y, específicamente, en aquellas áreas temáticas que se consideraban relevantes desde el punto de vista de las políticas culturales locales, a saber: (i) Cultura y desarrollo; (ii) Transversalidad de las políticas culturales; (iii) Cultura y participación ciudadana; (iv) Accesibilidad de la oferta cultural y (v) Papel de las iniciativas relacionadas con la memoria y la innovación en la 
construcción de la identidad local. Adicionalmente, debía incorporarse los logros que sobre sistemas de información y definición de indicadores se venían desarrollando por el grupo de trabajo en Cultura de Ciudades y Gobiernos Locales Unidos (CGLU) y de otras organizaciones, como el sistema de indicadores de Vigía-Observatorio Cultural de Cádiz ${ }^{2}$ o el Sistema de Información Cultural de CONACULTA. ${ }^{3}$

Por otro lado, el grupo de trabajo de la FEMP consideró desde un primer momento que los indicadores a considerar para formar parte del sistema debían ser indicadores confiables, útiles y conseguibles a nivel local (Dane, 2005; Cecchini, 2005); de forma que efectivamente permitiesen desarrollar un instrumento válido de seguimiento y evaluación, capaz de orientar la política cultural local y mejorar la toma de decisiones y la comparabilidad entre escenarios.

Con tal planteamiento, se adoptó una metodología de trabajo general (Dane, 2002; Castellanos, 2005), reproducible en cada una de los distintos ámbitos temáticos de interés, consistente a grandes rasgos en:

1. El establecimiento del marco teórico de referencia.

2. La definición de las necesidades de información a las que dar respuesta.

3. La exploración de potenciales indicadores.

4. El establecimiento de criterios de selección.

5. El proceso de selección y priorización de indicadores.

En palabras de Escudero (2009) -responsable en aquel entonces del Área de Cultura y Secretaria de la Comisión de Cultura de la FEMP, integrante del grupo de trabajo-, respecto de la selección de indicadores, cabe destacar que

La selección de indicadores se basaba en la verificación de si se había logrado o no una satisfactoria transformación de las necesidades de información en variables medibles, en la constatación de que la información que demandasen pudiese ser recolectada de forma útil y sostenible, y en la aplicación rigurosa de las exigencias o caracteres que un buen indicador debe reunir.

Estas características hacen referencia a ser medible, válido, fiable, comparable, robusto, relevante, claro y oportuno (Drucker, 1954; Bohner, 1979; Smith, 1999; Chapman, 2000; Schuster, 2001; Pfenniger, 2004; Iffacca, 2005; Carrasco, 2006; Jones, 2007).
Tras más de un año de intenso trabajo, a finales del mes de abril de 2007, se presentaba una versión preliminar de lo que tenía que constituir el Sistema de Indicadores.

Si bien el documento de trabajo fue extraordinariamente bien recibido, por cuanto ofrecía una metodología tanto teórica como práctica para facilitar a los gobiernos locales la evaluación de su política cultural; también era necesario reconocer que aún quedaba lejos de ser realmente una herramienta sencilla (y realmente útil para el propósito al que estaba destinada), dado el elevado número de indicadores que integraban el sistema diseñado, un total de 352.

Por esta razón, durante el primer semestre de 2008 se realizó una revisión exhaustiva de los indicadores propuestos, siendo clasificados en distintos niveles (Básico, Avanzado, Óptimo y Prescindible) en función no solo de la dificultad para su obtención sino también de la relación entre el esfuerzo que suponía su aplicación y la importancia de la información proporcionada por el mismo. Además, con esta clasificación se perseguía modular el sistema de forma que pudiese ser implantado por unas administraciones locales de manera secuencial, comenzando en principio por los indicadores relativamente más sencillos y continuando con los de nivel superior, avanzado y óptimo respectivamente; o alternativamente que fuese implantado por otras administraciones locales, por ejemplo, en base a programas.

Finalmente, en octubre de 2008 el sistema de indicadores diseñado fue presentado a la Comisión de Cultura de la FEMP y a la Dirección General de Política e Industrias Culturales; y en el año 2009 se publicó bajo el título Guía para la evaluación de las políticas culturales locales (FEMP, 2009).

\section{Hacia una herramienta para la evaluación de las políticas culturales locales. El Barómetro Cultural Local (BÁCULO)}

\section{Sistema de Información Cultural Local, SICLO}

La Guía para la evaluación de las políticas culturales locales elaborada por el grupo de trabajo de la FEMP constaba de un total de 317 indicadores, de los cuales 145 eran de naturaleza cualitativa y los restantes 172 de carácter cuantitativos. Los indicadores cualitativos 
esencialmente se vinculan con el ámbito de la autoevaluación y deben ser soportados por medio de evidencias documentales, en tanto que los indicadores cuantitativos se calculan a partir de los datos provenientes de fuentes diversas (estadísticas, registros administrativos, etc.) con el objetivo de mostrar una descripción de la realidad (Dunn, 2003; Baeker, 2002).

Sin embargo, para hacer efectivamente operativo el marco metodológico para el diseño de un sistema de indicadores culturales recogido en la Guía era necesario disponer de una aplicación tutelada que permitiese la recopilación, tratamiento y análisis de los datos necesarios para proporcionar a los gobiernos locales información útil y precisa con la finalidad de facilitar la evaluación de las políticas culturales. En un proyecto auspiciado por la FEMP y con el apoyo del Ministerio de Cultura, esta labor fue encomendada, a principios del año 2010, al grupo de investigación Métodos Cuantitativos para la Medición de la Cultura (MC2) de la Universidad de Valencia. La aplicación recibirá el nombre de SICLO, Sistema de Información Cultural Local (Coll et al., 2012).

El diseño y construcción del SICLO arrancó con la propuesta de indicadores contenidos en la Guía de evaluación de la FEMP; pero dado que el sistema originalmente estaba formado por un total de 317 , desde el principio se consideró que su dimensión era tan grande que resultaría inviable en la práctica. Por este motivo, se sometieron nuevamente los indicadores a un proceso de selección, basado en el análisis y consenso de un grupo de expertos formado por gestores culturales, académicos y técnicos de cultura tanto de la FEMP como de diversos ayuntamientos. Este proceso condujo a que finalmente se retuviesen para integrar el SICLO un total de 78 indicadores. La Figura 1 muestra la distribución del número de indicadores retenidos atendiendo al área de interés y su naturaleza (cuantitativos y cualitativos).

Reducida la dimensión del sistema, se realizó una prueba piloto (pretest) con el objetivo de contrastar la calidad de los cuestionarios confeccionados para obtener la información requerida, la calidad de los indicadores propuestos y recibir retroalimentación de los responsables de cultura de los gobiernos locales. Para ello se confeccionó un cuestionario telemático que fue cumplimentado por los usuarios potenciales del sistema.

Se pretendía que los participantes proporcionasen el mayor volumen posible de información para mejorar los contenidos del instrumento y su operatividad. Los gobiernos que finalmente participaron en la aplicación inicial contribuyeron aportando no solo los datos de que disponían sobre la cultura y la política cultural en la esfera local, sino también mediante numerosos comentarios y sugerencias relativos tanto a los cuestionarios que componían el instrumento como a los mecanismos de cumplimentación y administración de la información resultante. La recopilación de retroalimentación cualitativa sobre el sistema de indicadores resultó especialmente valiosa de cara a la calibración final del sistema de indicadores.

Actualmente se encuentra en funcionamiento el nivel básico de $\mathrm{SICLO}^{4}$ (www.mc2-uv.es/sido), que

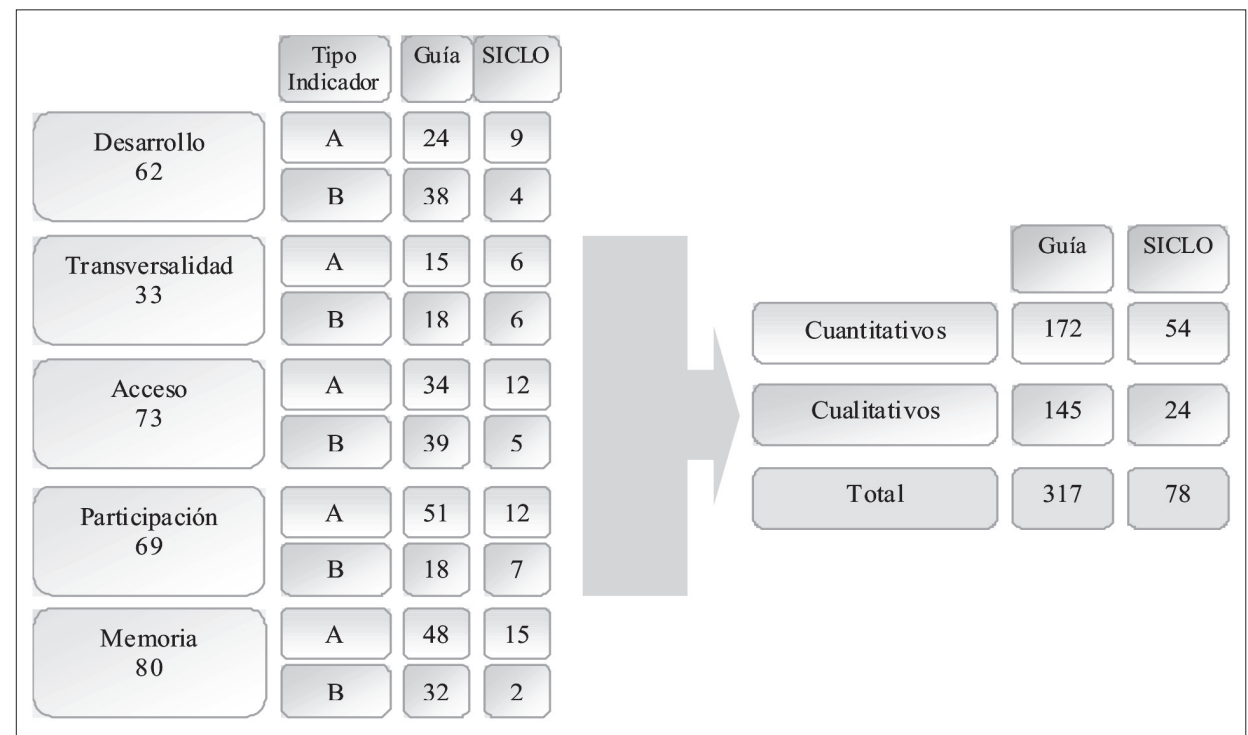

Figura 1. Distribución del número de indicadores. Comparativa guía de evaluación y SICLO.

Nota: A: Indicadores cuantitativos, B: Indicadores cualitativos

Fuente: COLL et al., 2012

4. El SICLO está modulado de la misma forma que la Guía para la evaluación de las políticas culturales en los niveles Básico, Intermedio y Avanzado. El nivel Básico, núcleo central del sistema, se encuentra integrado por un número limitado de indicadores básicos aplicables a todos los gobiernos locales. 
está integrado por un total de total de 18 indicadores cualitativos y 28 cuantitativos.

\section{Barómetro Cultural Local, BÁCULO}

La finalidad del BÁCULO es mejorar el conocimiento del estado de la cultura en el ámbito local y proporcionar información a los gobiernos locales (Ayuntamientos, Diputaciones, Cabildos) para apoyar tanto los procesos de planificación y toma de decisiones como el seguimiento y evaluación de las políticas culturales. Para conseguir esto, el BÁCULO, que se ha implementado sobre una plataforma interactiva web 2.0 (www.mc2-uv.es/Baculo), se alimenta de los datos almacenados en el módulo básico del SICLO, los analiza y sintetiza la información en un conjunto de indicadores sintéticos multidimensionales que pretenden reflejar la acción cultural del gobierno local.

Para cada gobierno local, BÁCULO proporciona información numérica y, fundamentalmente, gráfica sobre su posición relativa en cuanto a (i) la sintonía de la política aplicada con la Agenda 21 de la cultura (Indicador de Sintonía), (ii) la acción cultural (Indicador de Acción) y, por último, (iii) la intensidad del impulso cultural ejercido con relación a la respuesta cultural de la ciudadanía (Indicador de Impulso-Respuesta). Asimismo, para contextualizar el estado de la política cultural del gobierno local, se muestran los valores de una referencia contemporánea, que en el actual sistema está definida como el promedio de los gobiernos locales participantes. Sin embargo, a medi- da que se incremente la participación en el SICLO y el BÁCULO será factible definir referencias de comparación más específicas como, por ejemplo, el promedio de gobiernos locales con número de habitantes similar, con un esfuerzo cultural semejante o con un presupuesto de cultura similar.

\section{Indicador de Sintonía}

Mediante este indicador se pretende expresar el grado en que la política cultural aplicada por el gobierno local sintoniza con los postulados de la Agenda 21 de la Cultura respecto a los diversos ámbitos temáticos que esta contempla. De esta forma, este indicador contempla un total de 6 dimensiones ${ }^{5}$ que se corresponde con las siguientes áreas: a) cultura como factor de desarrollo, b) generación de tejido cultural local, c) transversalidad, d) fomento del acceso a la cultura, e) fomento de la participación ciudadana y f) apoyo a la memoria y a la creación. Para cada una de estas dimensiones, en el Cuadro 1 se muestra el tipo de información que se solicita a los usuarios para construir el indicador de Sintonía.

La información es recopilada a través de un cuestionario que se encuentra implementado en el módulo básico de SICLO ${ }^{6}$. El valor que el indicador de Sintonía toma en cada dimensión es el promedio de las respuestas a las preguntas contenidas en él. Como puede verse en la Figura 3, una vez tratados los datos, la información se presenta al usuario mediante

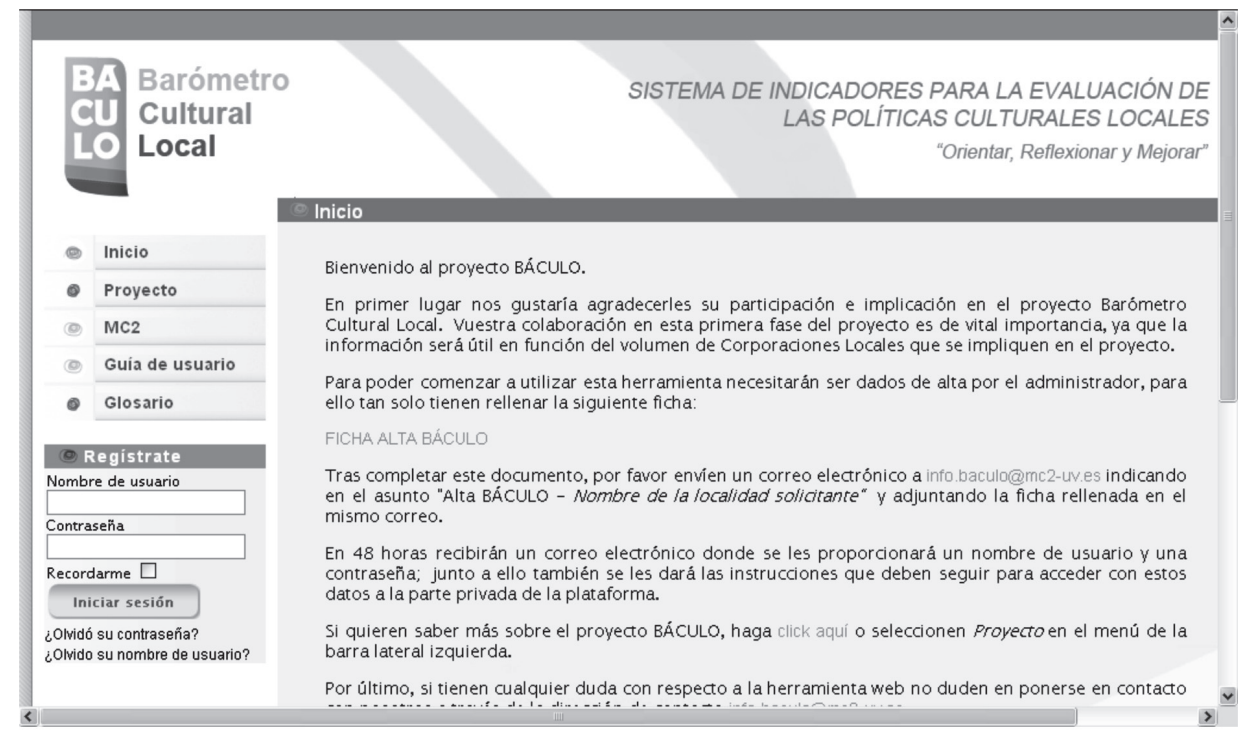

Figura 2. Página inicial de Báculo.

\footnotetext{
5. Estas dimensiones coinciden con las áreas temáticas en que se estructura el SICLO (Coll et al., 2012) solo que el área de Cultura y Desarrollo se ha subdividido en Cultura como factor de desarrollo sostenible y Cultura como factor de desarrollo del tejido cultural local.

6. Cabe la posibilidad de incorporar directamente los datos necesarios en el BÁCULO, puesto que dispone de acceso a los correspondientes cuestionarios de cumplimentación.
} 
Cuadro 1. Dimensiones del indicador de sintonía.

\section{La Cultura como factor de desarrollo sostenible}

1. Reconocimiento del potencial de contribución de la cultura a los objetivos de las políticas locales de desarrollo social, económico y urbano/territorial.

2.Amplitud en el establecimiento de objetivos de carácter cultural para las políticas locales en materia social, económica y urbano/territorial.

3. En qué medida las políticas culturales del gobierno local se dirigen expresamente a lograr objetivos de desarrollo social, económico y urbano/territorial.

La Cultura como factor de desarrollo del tejido cultural local

4. Existencia de censo de participantes o integrantes del tejido cultural local referido a empresas, entidades y profesionales.

5. Apoyo del gobierno local al desarrollo del tejido cultural a través de diferentes actuaciones, por ejemplo, sobre la creación, mantenimiento y consolidación de agentes del tejido cultural local.

6. Grado de reconocimiento de la cultura como fuente de sostenibilidad económica.

Transversalidad de la cultura

7. Presencia y reconocimiento de la cultura en las iniciativas y proyectos que son tratados desde la transversalidad y en el discurso del gobierno local como eje de desarrollo local sostenible.

8. Presencia de agentes culturales, internos y externos, en la definición de políticas económicas, territoriales y de ordenación y sociales del gobierno local.

9. Presencia de la cultura en las distintas políticas y áreas del gobierno local.

Acceso a la Cultura

10. En qué medida el gobierno local utiliza el factor precio como mecanismo para garantizar el acceso a la oferta cultural.

11. Grado en el que la comunicación es utilizada por el gobierno local para garantizar el acceso a la oferta cultural.

12. En qué medida el gobierno local fomenta la multiculturalidad, la interculturalidad y la monoculturalidad para garantizar el acceso a la oferta cultural.

Cultura y participación ciudadana

13. Grado de disponibilidad de inventarios de equipamientos culturales.

14. Nivel de desarrollo de sistemas de recogida de información de la oferta cultural local.

15. Existencia de diversidad de canales para que los ciudadanos puedan participar y valorar el diseño de la cultura local.

Papel de la memoria e innovación en la creación de la identidad local

16. Medios mediante los que el gobierno local apoya el patrimonio cultural y otras manifestaciones de la memoria colectiva.

17. Mecanismos a través de los cuales se establece la intención del gobierno local para impulsar la creación cultural como seña de identidad.

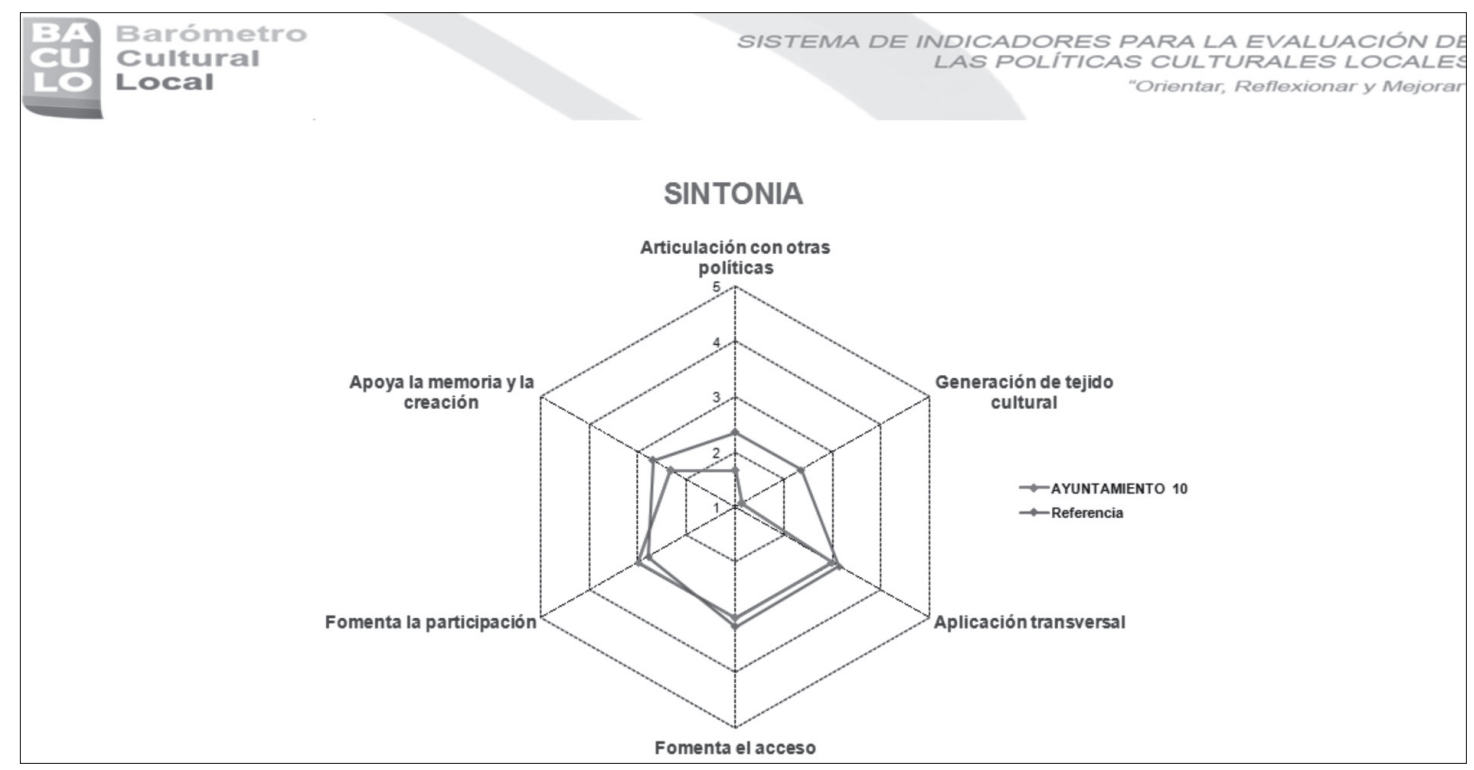

Figura 3. Indicador de Sintonía. 
un gráfico de tipo radial en el que cada uno de los ejes corresponde a cada una de las áreas temáticas o dimensiones que contempla el indicador. Para cada dimensión se representa tanto la posición relativa del gobierno local que efectúa la consulta como la posición que ocupa la referencia.

De esta forma, al comparar su posición con respecto a la referencia, el usuario puede determinar si la sintonía de su política cultural es mayor, similar o menor para cada una de las áreas temáticas. También es posible consultar los resultados concretos obtenidos en cada una de las áreas temáticas. Esta información también se muestra por medio de un gráfico radial, solo que en esta ocasión los ejes vienen representados por los ítems que se preguntan en cada dimensión.

\section{Indicador de Acción}

Describe la intervención del gobierno local en términos de los recursos destinados a la cultura y de los diversos canales y mecanismos que utiliza para su aplicación. El indicador contempla cinco dimensiones: presupuesto, personal, dotaciones, equipamientos y actividades, y está basado en las respuestas de los gobiernos locales participantes a un cuestionario cuantitativo. En el Cuadro 2 se resumen, en términos generales, las medidas que se abordan en cada dimensión del indicador de acción.

Para analizar cada dimensión del indicador de acción se cuenta con un conjunto de preguntas que vienen expresadas, en general, en escalas y unidades distintas. Evidentemente, esto supone una dificultad añadida a la hora de obtener un único valor que represente la acción del gobierno en cada una de las cinco dimensiones. Para salvar este obstáculo la decisión que se adoptó consistió en (i) relativizar los valores numéricos de cada medida en función del número de habitantes, (ii) obtener los quintiles de la distribución de frecuencias de las medidas relativizadas y, a continuación, (iii) asignar los valores de 1 a 5 según el quintil al que pertenece la respuesta relativizada de un gobierno local en particular. La puntuación del indicador en cada dimensión será el promedio de los valores así asignados a las cuestiones que lo integran.

El feedback que se proporciona al usuario es doble. Por una parte, como se muestra en la Figura 4, cada una de las dimensiones del indicador de Acción se colorea atendiendo al desempeño del gobierno local en relación con la referencia: verde (superior), amarillo (similar) y rojo (inferior).

Por otra parte, y como sucediera con el indicador de Sintonía, el indicador de Acción se ilustra mediante un gráfico radial (Figura 5). En los ejes se representan las cinco dimensiones de las que consta
Cuadro 2. Dimensiones y medidas del indicador de acción.

Presupuestos

1. Presupuesto del área de cultura.

2. Presupuesto de gasto local en cultura.

3. Presupuesto destinado a apoyar el tejido cultural local.

4. Proporción del presupuesto total destinado a proyectos culturales con tratamiento transversal.

5. Presupuesto destinado a la investigación, protección, mantenimiento y socialización/difusión de la memoria cultural material e inmaterial local.

6. Porcentaje del presupuesto cultural destinado a iniciativas de socialización/difusión de la creación cultural y los nuevos lenguajes creativos.

Personal, trabajadores del gobierno local dedicados a:

7. Programación cultural.

8. Difusión cultural.

9. Equipamientos culturales

Dotaciones

10. $\mathrm{N}^{\mathrm{o}}$ de bienes de interés cultural y otros bienes inventariados.

11. $N^{o}$ de museos, centros de documentación, centros de interpretación del patrimonio, etc.

12. $\mathrm{Km}^{2}$ de superficie de espacios naturales protegidos.

\section{Equipamientos}

13. Porcentaje de equipamientos que incorporan diversos elementos de confort para usuarios.

14. $\mathrm{N}^{\mathrm{o}}$ de butacas en teatros, auditorios, salas polivalentes, etc.

15. $\mathrm{m}^{2}$ de espacios para formación cultural y práctica artística.

16. $\mathrm{N}^{\circ}$ de puestos de lectura/consulta en bibliotecas.

\section{Actividades}

17. $\mathrm{N}^{\mathrm{o}}$ de iniciativas o proyectos culturales.

18. $\mathrm{N}^{\mathrm{o}}$ de proyectos transversales de naturaleza cultural.

19. $\mathrm{N}^{\mathrm{o}}$ de actividades culturales organizadas por precio.

20. Distribución de actividades culturales por meses del año.

21. $\mathrm{N}^{\mathrm{o}}$ de actividades culturales destinadas a público familiar.

22. $\mathrm{N}^{\mathrm{o}}$ de actividades culturales para escolares.

23. $\mathrm{N}^{\mathrm{o}}$ de entradas vendidas para actividades culturales.

24. $\mathrm{N}^{\mathrm{o}}$ de asistentes a los acontecimientos culturales gratuitos.

25. $\mathrm{N}^{\mathrm{o}}$ de asistentes a las actividades culturales por contenidos y segmentos de público.

26. $\mathrm{N}^{\mathrm{o}}$ de beneficiarios de actividades pedagógicas sobre el patrimonio.

27. $\mathrm{N}^{\circ}$ de visitantes a museos. 
el indicador y los valores se refieren a las posiciones relativas que ocupan tanto el gobierno local como la referencia de comparación.

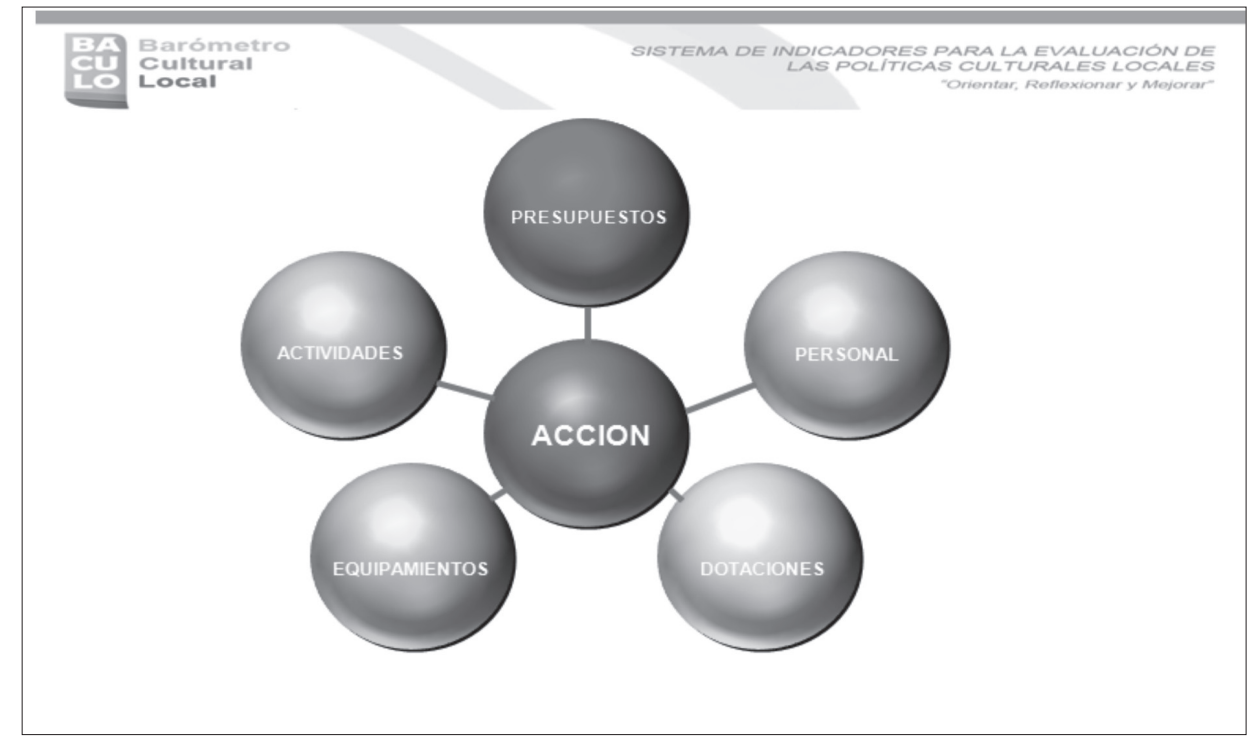

Figura 4. Indicador de Acción y sus dimensiones.

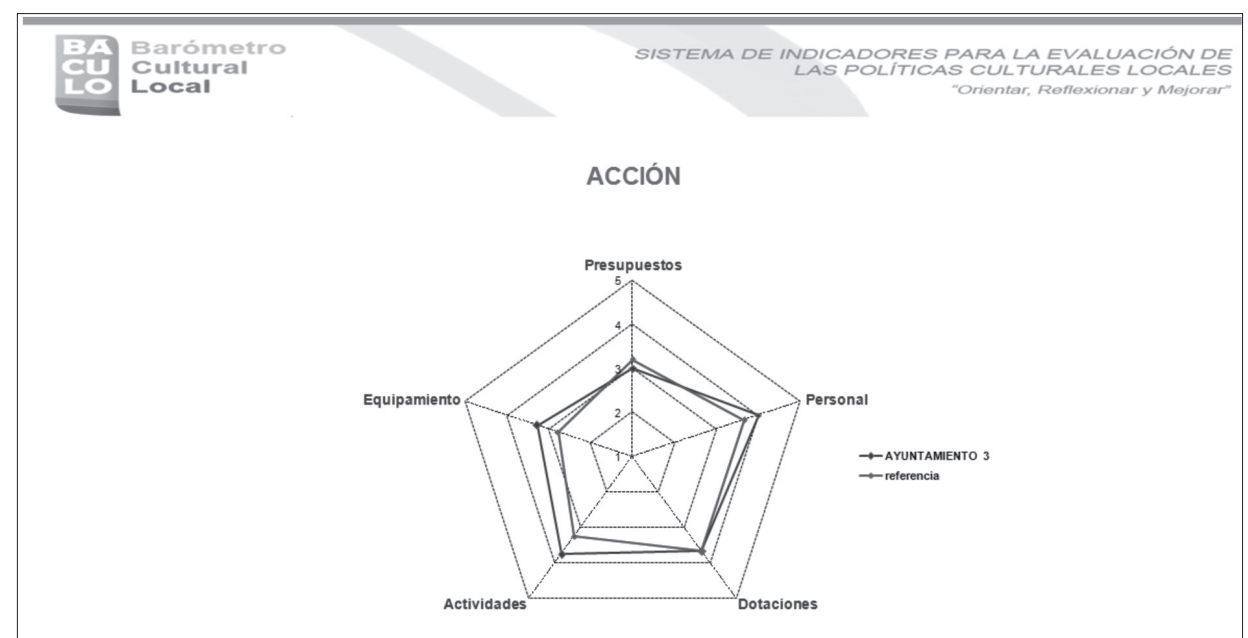

Figura 5. Indicador de Acción: comparación posición gobierno local y referencia.

\section{Indicador de Impulso-Respuesta}

Por último, con el indicador de Impulso-Respuesta se persigue evaluar la relación que existe entre el impulso cultural ejercido por el gobierno local y la respuesta ciudadana en forma de demanda cultural. La definición dual del indicador requiere, como paso previo, calcular índices adecuados para reflejar ambos aspectos de la realidad, impulso y respuesta, y poderlos confrontar entre sí.

Con esta finalidad, el impulso ejercido por la política cultural local es evaluado en términos (i) de las dotaciones y equipamientos gestionados por el gobierno local y (ii) de los proyectos y actividades cul- turales programados, organizados o apoyados desde el gobierno local. En el Cuadro 3 se recogen las medidas que son consideradas para calcular el indicador de impulso, relativizadas en función del presupuesto destinado a cultura por el gobierno local.

El estado contemporáneo de la respuesta ciudadana, por su parte, es evaluado en términos (i) de la utilización que la ciudadanía hace de los servicios culturales provistos por el gobierno local y (ii) de la participación individual y colectiva en las actividades culturales programadas, organizadas o apoyadas por el gobierno local. Las medidas que se reflejan en 
el Cuadro 4, relativizadas en función del número de habitantes, son las empleadas para determinar el indicador de respuesta.

Cuadro 3. Medidas para calcular el indicador de impulso.

\begin{tabular}{|l|}
\hline Dotaciones y equipamientos \\
1. $\mathrm{N}^{\circ}$ de butacas \\
2. $\mathrm{N}^{\circ}$ de museos \\
3. $\mathrm{N}^{\circ}$ de puestos de lectura \\
4. $\mathrm{m}^{2}$ espacios dedicados a actividades de formación \\
cultural y prácticas artísticas \\
\hline Actividades promovidas \\
5. Actividades gratuitas \\
6. Actividades de pago \\
7.Actividades para escolares \\
8. Actividades público familiar \\
\hline
\end{tabular}

Cuadro 4. Medidas para calcular el indicador de respuesta.

\begin{tabular}{|l|}
\hline Utilización de servicios \\
1.Visitas a la web \\
2. Asistentes gratuitos \\
3.Visitantes a museos \\
4. $\mathrm{N}^{\mathrm{o}}$ de usuarios de bibliotecas \\
\hline Participación en la vida cultural \\
5. $\mathrm{N}^{\circ}$ de beneficiarios de formación \\
6. $\mathrm{N}^{\circ}$ de creativos afincados \\
7. $\mathrm{N}^{\circ}$ asociaciones \\
8. $\mathrm{N}^{\circ}$ de entradas vendidas \\
\hline
\end{tabular}

Los índices de Impulso (por euro gastado) y Respuesta (por habitante) son la media de los valores de las componentes que los integran, que a su vez se calculan siguiendo el mismo procedimiento que el descrito para el indicador de Acción. Las bases de comparación son los promedios del conjunto de gobiernos participantes que se fijan en el 100\%. Respecto de este se rescalan los indicadores de Impulso-Respuesta. Los resultados obtenidos son presentados a los usuarios por medio de dos gráficas. En la primera de ellas (Figura 6) se relacionan las distintas componentes del indicador que se analiza. Como puede verse a través de un ejemplo, el indicador de Impulso es 150,6 y el de Respuesta es 190,05. Esto significa que el impulso ejercido a través de su política cultural por el gobierno local es un 50,6\% superior a la media, siendo la respuesta de los ciudadanos un 90,05\% mayor que el de la referencia, es decir, la respuesta es proporcionalmente más intensa que el impulso realizado.

En la segunda gráfica (Figura 7) se representa un diagrama de dispersión con los valores de los indicadores de Impulso-Respuesta de los gobiernos locales participantes. El origen del eje de coordenadas del diagrama de dispersión (eje de abscisas: indicador de Impulso y eje de ordenadas: indicador de Respuesta) viene determinado por los valores de la referencia. Se destaca en color rojo la posición del gobierno local que consulta sus resultados, facilitando así la comparación con el resto de gobiernos.

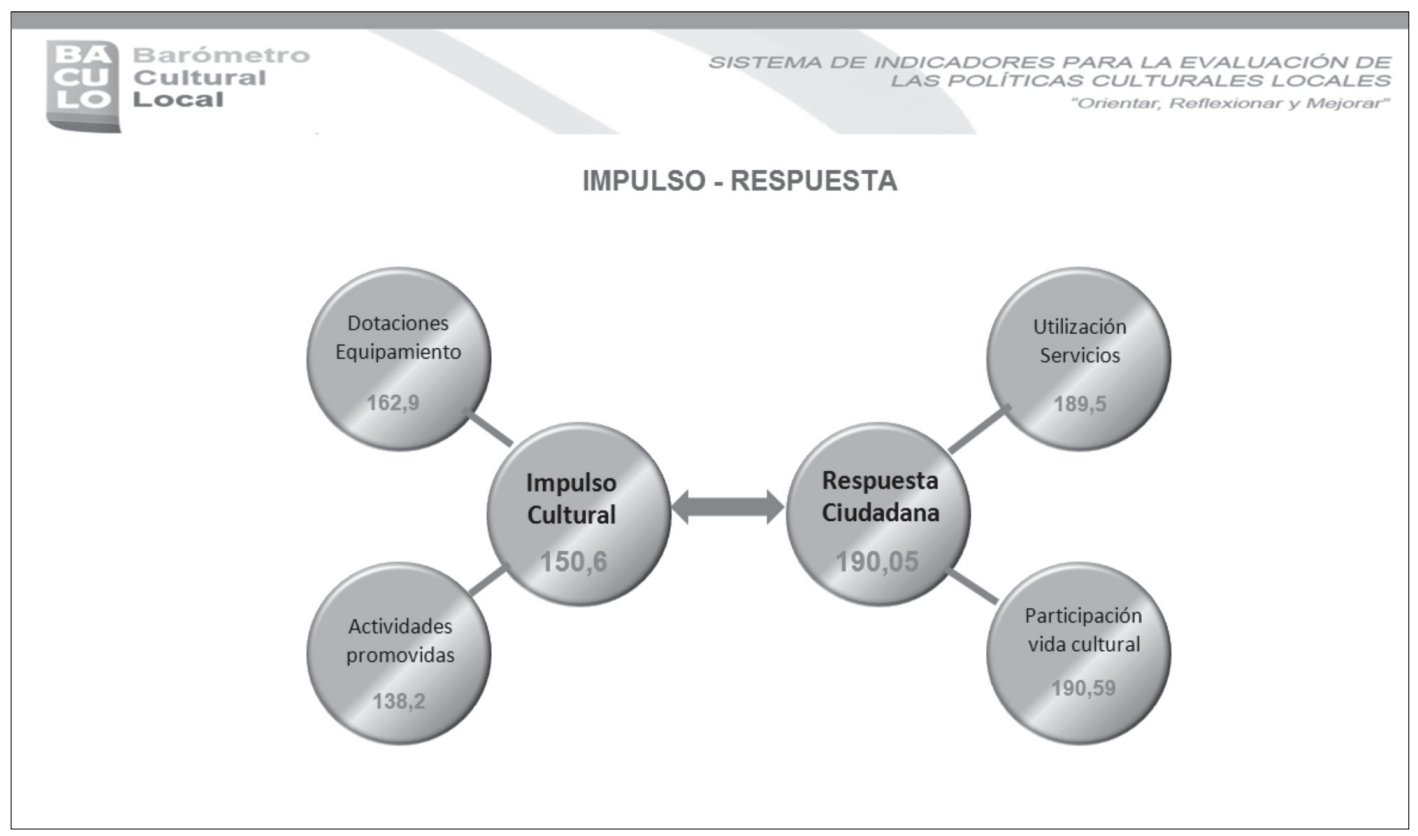

Figura 6. Indicador de Impulso-Respuesta y relación con sus componentes. 


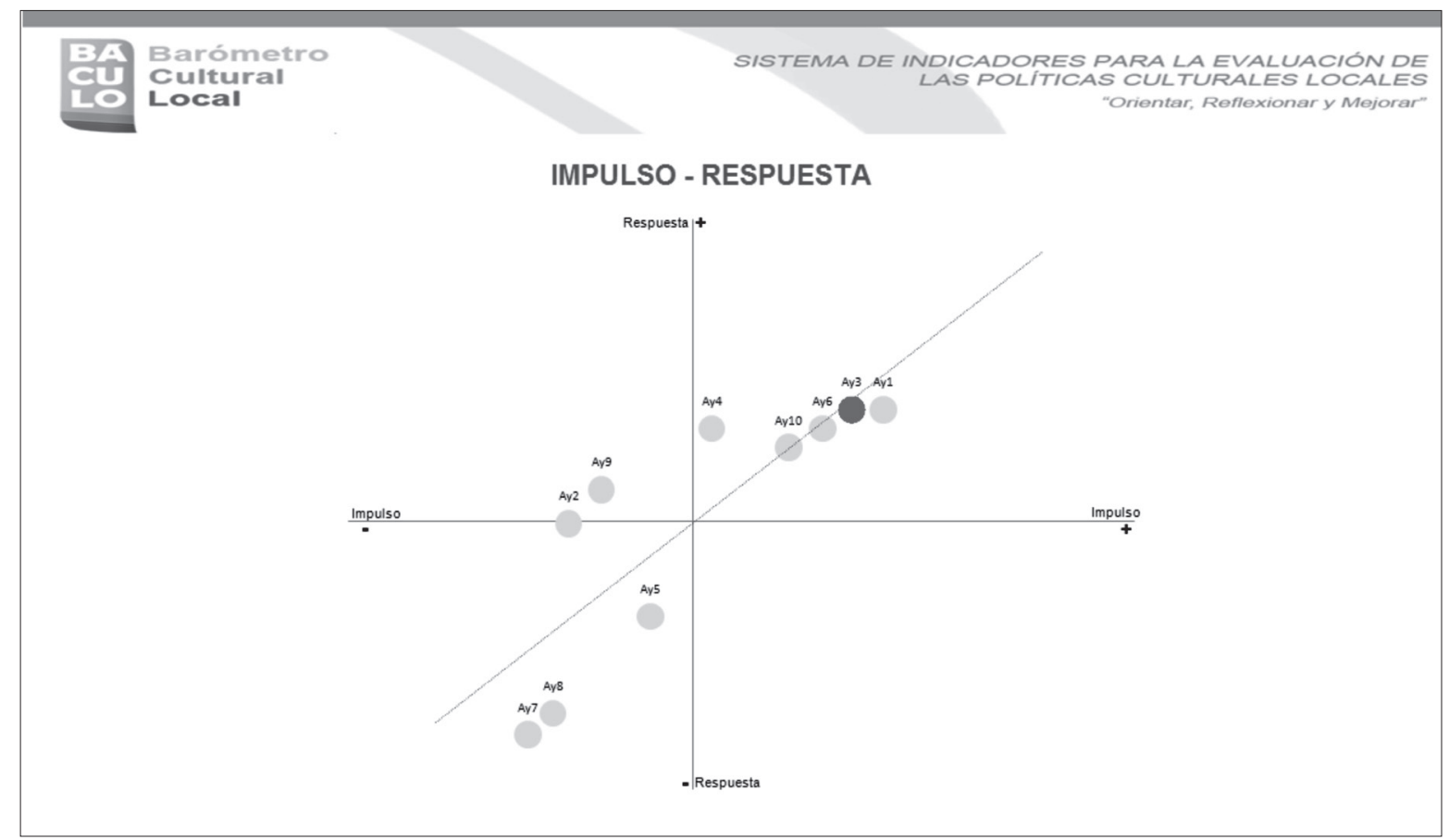

Figura 7. Diagrama de dispersión del indicador Impulso-Respuesta.

\section{Consideraciones finales}

El Barómetro Cultural Local (BÁCULO) es una herramienta que proporciona información a los gobiernos locales para apoyar tanto los procesos de planificación y toma de decisiones como el seguimiento y evaluación de las políticas culturales. Para lograrlo, a partir de los datos contenidos en el Sistema de Indicadores Culturales Local (SICLO), el BÁCULO construye tres indicadores sintéticos multidimensionales. El indicador de Sintonía, que pretende determinar el grado en que la política cultural aplicada por el gobierno local sintoniza con los postulados de la Agenda 21 de la Cultura; el indicador de Acción, que describe la intervención del gobierno local y, finalmente, el indicador de Impulso-Respuesta, que persigue evaluar la relación que existe entre el impulso cultural ejercido por el gobierno local y la respuesta ciudadana en forma de demanda cultural.

La información que BÁCULO proporciona a los gobiernos locales participantes es fácilmente interpretable puesto que fundamentalmente se basa en representaciones gráficas. Se trata de que el usuario pueda comparar visualmente su posición con la de una referencia, constituida por el promedio de los valores de los gobiernos locales participantes. El resultado de la comparación permitirá a cada gobierno local identificar si la orientación, el desempeño y el rendimiento de su política cultural son superiores, similares o inferiores a la de la referencia, lo que sin duda puede orientar y facilitar el proceso de mejora.

La labor que queda pendiente es la referida a la divulgación y comunicación de las potencialidades del BÁCULO. Como ya sucediera con el SICLO, el mecanismo que se considera más adecuado para difundir esta nueva herramienta es a través de talleres de formación en la FEMP. Con estas acciones formativas se pretende diseminar la experiencia, fidelizar a los participantes y, sobre todo, atraer a nuevos gobiernos locales para que se incorporen al sistema. Cuanto mayor sea la participación mayor será la disponibilidad de información, lo que facilitará la construcción de referencias de comparación más específicas y dotará de mayor precisión y calidad a la información que se obtenga. 


\section{Referencias}

BAEKER, G. Measures and Indicators in Local Cultural Development. Toronto: EUCLID, 2002.

BOHNER, L. Indicators of cultural development within the European context. Current surveys and research in statistics. Cap. 10. Working paper ST-79/Cof 602/92. UNESCO, 1979.

CARRASCO ARROYO, S. Medir la cultura, una tarea inacabada. Periférica, s.1., n. 7, p. 140-168, 2006.

CASTELlANOS, A. Asistencia técnica para la elaboración de un diagnóstico y una metodología para diseñar indicadores culturales en los países centroamericanos. Informe final, UNESCO, 2005.

CECCHINI, S. Indicadores sociales en América Latina y el Caribe. Serie de Estudios Estadísticos y Prospectivos CEPAL, Chile, n. 34, 2005.

CHAPMAN, A. Indicators and Standards for Monitoring Economic, Social and Cultural Rights. In: SECOND GLOBAL FORUM ON HUMAN DEVELOPMENT. Universidade Cândido Mendes, Rio de Janeiro, 2000. CIUDADES Y GOBIERNOS LOCALES UNIDOS, CLGU. Agenda 21 for Culture. United Cities and Local Governments - Committee on culture, 2004. Disponible en: http://agenda21culture.net Acceso: 15 octubre 2012.

COLL-SERRANO, V.; CARRASCO-ARROYO, S.; BLASCO-BLASCO, O.; VILA-LLADOSA, L. Sistema de indicadores culturales locales (SICLO). El Profesional de la Información, v.21, n.1, p. 113-117, 2012.

DANE, DEPARTAMENTO ADMINISTRATIVO NACIONAL DE ESTADÍSTICA. Elementos metodológicos básicos para la selección, construcción, interpretación y análisis de indicadores. Bogotá: Sistema Nacional de Información Estadística (SNIE), 2002.

Guía para diseño, construcción e interpretación de indicadores, 2005. Disponible en: http://www.dane.gov. co/files/planificacion/fortalecimiento/cuadernillo/Guia_ construccion_interpretacion_indicadores.pdf Acceso: 15 octubre 2012.

DRUCKER, P. The Practice of Management. Harper and Row: New York, 1954.
DUNN, W. Public Policy Analysis: An Introduction. 4ª ed. Upper Saddle River, NJ: Prentice Hall, 2003.

ESCUDERO MÉNDEZ, J. La construcción del sistema de indicadores para la evaluación de las políticas culturales locales desarrollado por la FEMP. Historia de un proceso de cooperación. Periférica, s.1., n. 9, p. 115-132, 2008.

EVANS, G. Cultural planning, an urban renaissance? London: Routledge, 2001.

FEMP. Guía para la evaluación de políticas culturales locales. Madrid: Federación Española de Municipios y Provincias, 2009.

IFFACCA, INTERNATIONAL FEDERATION OF ARTS COUNCILS AND CULTURE AGENCIES. Statistical Indicators for Arts Policy. Sidney: IFFACCA, 2005. JONES, R. Project management survival: a practical guide to leading, managing and delivering challenging projects. London: Kogan Page Limited, 2007.

MARTINELL, A. Evaluación en gestión y políticas culturales. Entre el deseo y la realidad. In: CONFERENCIA CULTURA Y DESARROLLO. DISEÑO DE INDICADORES. Secretaría de Cultura del Distrito Federal, México, 2011.

MUSSOT, M. L. Relevancia de la evaluación de las políticas públicas. In: CONFERENCIA CULTURA Y DESARROLLO. DISEÑO DE INDICADORES. Secretaría de Cultura del Distrito Federal, México, 2011. PFENNIGER, M. Indicadores y estadísticas culturales: un breve repaso conceptual. Portal Iberoamericano de Gestión Cultural, España, 2004. Disponible en: http://www. gestioncultural.org/articulos.php?id_documento $=302150$ Acceso: 15 octubre 2012.

SCHUSTER, J. On performance indicators. In: (Ed.). Policy and Planning with a Purpose of The Art of Making Choices in Arts Funding. Chicago: The Cultural Policy. Center at the University of Chicago, 2001.

SMITH, D. K. Make Success Measurable!: A MindbookWorkbook for setting goals and taking action. New York: John Wiley and Sons, Inc. 1999.

\section{O barômetro cultural local (báculo): uma ferramenta para acompanhamento e avaliação de políticas culturais locais}

\section{Resumo}

O Barômetro Cultural Local (Báculo) é uma ferramenta que proporciona informação aos governos locais para apoiar tanto os processos de planejamento e de tomada de decisão quanto o acompanhamento e a avaliação das políticas culturais. Partindo dos dados contidos no Sistema de Indicadores Culturales Local (SICLO), a informação que o Báculo oferece aos usuários é sintetizada em três indicadores multidimensionais: sintonia, ação e impulso-resposta. Neste artigo são descritas as características principais do Báculo, quer dizer, como se obtêm os indicadores que o integram e como a informação é apresentada aos governos locais.

Palavras-chave: sistema de indicadores, indicadores culturais, política cultural, governo local e municipal, planejamento, tomada de decisões. 


\title{
Local cultural barometer (báculo): a tool for monitoring and evaluation of the local cultural policies
}

\begin{abstract}
The Local Cultural Barometer (BÁCULO) is a tool whose aim is (i) to provide information to the local governments to support the process of planning and decision making, and (ii) to facilitate the follow-up and evaluation of the cultural policies. From the data contained in the Local Cultural Indicators System (SICLO), the information that BÁCULO offers to the users is synthesized in three multidimensional indicators: Tuning, Action and Impulse-Response. This paper describes BÁCULO's main characteristics, that is to say, how the different indicators are obtained and how the information is shown to the local governments.
\end{abstract}

Key words: Indicators Systems, cultural indicator, local and municipal government, planning, decision making.

Data de recebimento do artigo: 28/10/2012

Data de aprovação do artigo: 11/04/2013 